Family Medicine and Community Health

\title{
Antimicrobial dispensing practices and determinants of antimicrobial resistance: a qualitative study among community pharmacists in Pakistan
}

\author{
Zikria Saleem (D) , ${ }^{1}$ Mohamed Azmi Hassali, ${ }^{1}$ Furqan Khurshid Hashmi, ${ }^{2}$ \\ Brian Godman, ${ }^{3}$ Fahad Saleem ${ }^{4}$
}

To cite: Saleem Z, Hassali MA, Hashmi FK, et al. Antimicrobial dispensing practices and determinants of antimicrobial resistance: a qualitative study among community pharmacists in Pakistan. Fam Med Com Health 2019;7:e000138. doi:10.1136/fmch-2019-000138

- Additional material is published online only. To view please visit the journal online (http://dx.doi.org/10.1136/fmch2019-000138).

Received 12 March 2019 Revised 10 June 2019 Accepted 13 June 2019

Check for updates

(C) Author(s) (or their employer(s)) 2019. Re-use permitted under CC BY-NC. No commercial re-use. See rights and permissions. Published by BMJ.

${ }^{1}$ School of Pharmaceutical Sciences, Universiti Sains Malaysia, Penang, Malaysia ${ }^{2}$ University College of Pharmacy, University of the Punjab, Lahore, Pakistan

${ }^{3}$ Strathclyde Institute of Pharmacy and Biomedical Sciences, Strathclyde University, Glasgow, UK

${ }^{4}$ Faculty of Pharmacy and Health sciences, University of Balochistan, Quetta, Pakistan

Correspondence to

Dr Zikria Saleem;

xikria@gmail.com

\section{ABSTRACT}

Background There are concerns with the extent of dispensing of antibiotics among community pharmacists in Pakistan often without a prescription adding to antimicrobial resistance (AMR) rates.

Objective To explore the determinants of AMR and the pattern of antimicrobial dispensing among community pharmacists.

Design In this qualitative study design, a semistructured interview guide was developed based on an in-depth review of published papers. Audio-recorded interviews with transcripts were analysed by thematic content analysis.

Setting Interviews were conducted among community pharmacists in Lahore, Pakistan.

Participants In order to obtain individual points of view, in-depth face-to-face interviews with purposively selected pharmacists were conducted.

Results A total of 12 pharmacists were interviewed for the study. After analysis, four major themes emerged: (1) knowledge and perception of community pharmacists about antimicrobials, (2) antimicrobial dispensing practices of community pharmacists, (3) determinants of AMR, (4) potential interventions to control AMR. Most of the pharmacists have limited knowledge about AMR, antimicrobial stewardship programmes and related guidelines. However, all the pharmacists strongly agreed that different appropriate actions should be taken in order to rationalise future antimicrobial use.

Conclusion The results indicated that irrational antimicrobial dispensing and use is common among community pharmacists in Pakistan owing to lack of knowledge. The community pharmacists perceived that behaviour of patients and the societal environment contributed to irrational antimicrobial use and subsequent development of AMR. They suggested a need for a multidisciplinary framework in order to improve future antimicrobial use and reduce AMR in Pakistan.

\section{INTRODUCTION}

There are concerns with rising rates of $\mathrm{AMR}$, leading to urgent calls by the WHO, UN and others to address this including the development of country-specific plans National Action Plans on AMR such as the National

\section{Key points}

- Antimicrobial resistance (AMR) is a major concern in Pakistan worsened by inappropriate antibiotic use.

- The government of Pakistan has recently launched their national action plan to reduce AMR through enhancing appropriate antibiotic use.

- There were concerns with the knowledge of community pharmacists about AMR and practices of inappropriate dispensing of antibiotics.

- The study emphasised the need to introduce a number of measures to improve the future use of antibiotic.

Action Plan of Pakistan. ${ }^{1}$ Otherwise, AMR rates will grow appreciably impacting on morbidity, mortality and costs. ${ }^{23}$ This is no longer a prediction for the future but is already happening across the world, especially in developing countries. ${ }^{4}$ Several modifiable factors are accountable for increasing AMR rates. ${ }^{5}$ The overprescribing of antimicrobials for acute respiratory infections in ambulatory care, which are typically viral in origin, is one contributing factor enhanced by pressure from patients. ${ }^{6}$ Selling antimicrobials without prescriptions is also common in many lower-income and middle-income countries (LMICs), as well as more common in Eastern versus Western European countries, ${ }^{7}$ adding to AMR rates, especially if antibiotics are dispensed for viral infections such as coughs and colds. Overall, the determinants that are seen to contribute to the widespread dispensing of antibiotics without a prescription include poor enforcement of legislation, lack of knowledge among pharmacist about infectious disease, pressure of customers, the business nature of pharmacy and monetary incentives from pharmaceutical companies. ${ }^{8-10}$ In addition, overprescribing of antibiotics by physicians for predominantly 
viral infections adds to increasing AMR rates, ${ }^{6}$ with patients believing this represents normal care and consequently they believe it is acceptable to ask pharmacists in the future for antibiotics without necessarily seeking physician help, especially where affordability is an issue. Moreover, the natural and physical environment also has a great impact on antimicrobial use behaviour and subsequently on AMR. ${ }^{11}$

With respect to healthcare, community pharmacists are often the first healthcare professional patients see about their condition and consequently play a key role in subsequent patient management. ${ }^{8}{ }^{12} 13$ In addition, pharmacies typically stay open later than ambulatory care clinics and are generally more accessible than physicians, which is welcomed by patients, especially if they cannot afford to take time off work to visit a busy clinic. ${ }^{1415}$ In addition, in a number of LMICs, patients cannot afford to see both a physician and purchase any medicine. This is leading to calls to improve the education of pharmacists about the aetiology of common infections, side-effects of antimicrobials and appropriate dispensing of antimicrobials, starting in pharmacy schools and continuing with the production of appropriate guidelines for pharmacists, which is beginning to happen and will continue. ${ }^{16}$ We are aware of the high rates of self-purchasing of antimicrobials and other medicines in Pakistan often for economic reasons as well as ease of access. ${ }^{17-19}$ However, in Pakistan, community pharmacists are facing new challenges to attract and retain customers as more and more pharmacies are being established, which is making community pharmacy more competitive. As a result, we believe community pharmacists are dispensing antimicrobials without prescription in a number of situations in order to nurture the loyalty of customers and to prevent losing them to competitors. We also believe patients are taking advantage of this where this happens by influencing and pressurising community pharmacists to dispense antimicrobials of their choice without a legitimate prescription from physicians often for self-limiting conditions. ${ }^{12} 20$

We are aware that a number of qualitative studies have already been conducted to explore the knowledge, perception and practices of healthcare professionals, especially pharmacists about antimicrobial sales without a prescription, their use, development of AMR as well as the instigation of antimicrobial stewardship programmes (ASP). ${ }^{10-12} 2122$ However, to the best of our knowledge, no such study has been reported from Pakistan. Consequently, in the first instance, the purpose of this qualitative study was to explore the determinants of AMR and the patterns of antimicrobial dispensing among community pharmacists at Lahore, Pakistan. At this stage in the research, the study will provide future guidance to help implement the national action plan of Pakistan to reduce AMR. This is because we are aware, as mentioned, of the increasing importance of community pharmacists in Pakistan in the management of self-limiting conditions such as coughs and colds.

\section{METHODS}

\section{Study design}

A qualitative study design was adopted for this study. This is because a qualitative research design is considered to be the most appropriate option to explore a wide range of in-depth viewpoints and extract key information and ideas from healthcare professionals. The consolidated criteria for reporting qualitative research (COREQ) checklist for reporting qualitative studies guided the reporting of the methods and the results. ${ }^{23}$

\section{Instrument development}

A semistructured interview guide (online supplementary annexure 1) was developed based on an in-depth review of the published literature and the knowledge of the co-authors who have worked extensively in this area. ${ }^{12-15} 2122$ 24-27 Before conducting the interviews, the interview guide was assessed for its validity and reliability. By applying cumulative and argumentative techniques, experts from the Universiti Sains Malaysia, University of Balochistan and the University of the Punjab (MAH, FS, FKH) with experience in the relevant field validated the guide before use. All the questions were also reviewed and approved by two independent pharmacists working in community pharmacies to enhance the applicability and robustness of the interview guide. A pilot study was also conducted before the formal interviews, and the guide was improved accordingly to further enhance the robustness of the findings. The reliability of the study findings was assured by keeping records of face-to-face interviews with the community pharmacists.

\section{Respondents sampling and inclusion criteria}

The study was conducted in Lahore, the capital of the province of Punjab, which is the second largest and most populous city of Pakistan. Lahore is a metropolitan city with a population of approximately 10 million. Currently, the community pharmacy setup of Lahore is undergoing both evolutionary and revolutionary changes. The total number of registered and licensed pharmacies in Lahore was 3618 in 2016, and one pharmacist is mandatory per premise in order for the premise to obtain a license to dispense medicines. Registered pharmacists with the Punjab Pharmacy Council (PPC) were subsequently included by using a purposive sampling technique. Overall, peer pharmacists were included who were working in licensed pharmacies and had a license issued by the Director General Health Services, Department of Health, Government of the Punjab, Lahore, Pakistan.

\section{In-depth face-to-face interviews}

Before the interview, the pharmacists participating in the study were verbally informed about the objective of the study. Written consent was obtained from the study respondents for their interviews to be audio-recorded. They were also allowed to voluntarily withdraw themselves from the study at any point. The interviews were conducted one on one at the place chosen by the 
interviewee (office, residence, clinic, hospital). The first part of each interview was related to personal and professional information of the pharmacist such as their age, college attended, year of graduation, working organisation and length of experience. The second part of the interview related to their knowledge and attitudes towards antimicrobials use. In the third part, their perception about antimicrobial use and resistance was explored. In the fourth part, questions were asked relating to their practical experience regarding antimicrobial dispensing practices, counselling habits, frequency of customers asking for antimicrobials and other related experiences. In the final part, six hypothetical case scenarios based on realistic incidents were discussed to simulate situations pertinent to the practice of the interviewees. All interviews were conducted in English. Each interview lasted for 30-45 min. Probing questions were also used and interviewees were given liberty to describe additional views and comments. All interviews were audio-recorded, and additional field notes were also taken by the experienced principal researcher ZS (male) who is a Ph.D. scholar. Interviews were conducted until the occurrence of saturation, a point at which no new data emerge. Due to the time constraints, three pharmacists refused to participate in the study. Saturation was attained by the 10th interview; however, two more interviews were conducted to ensure saturation. The interviews were conducted between October 2017 and January 2018.

\section{Analysis}

Audio-recorded interviews were transcribed verbatim. In the process of data extraction, the verbatim speech has been minimally changed for grammatical errors. In the first step of data analysis, a thematic content analysis (TCA) was performed in which relevant answers from each of the transcripts relating to the general research questions were deductively highlighted. In the second step, one respondent's answers were compared and contrasted with those from another respondent to ascertain any differences. The identified patterns were then reorganised and emerging themes were identified from the interview transcripts. The analysis provided a chance to establish initial themes and then look for divergent or negative responses, exploring our interpretations and the complexity of the data. The ideas were discussed within the research team during the course of the interviews in order to explore pertinent issues further. We constantly sought to keep the richness of the pharmacists' experiences, noting atypical cases, contradictions and conflicts within the data. On categorising a theme, we explored through all the transcribed interviews for interrelated comments. This practice ensured cases initially observed as unrelated could be clustered together as their interconnectedness became apparent.

\section{Ethics statement}

The study was approved by the Human Ethics Committee for Clinical Research of the Punjab University College
Table 1 Demographics of respondent community pharmacists

\begin{tabular}{llc}
\hline Demographics & N & $\%$ \\
\hline Gender & & \\
$\quad$ Male & 11 & 91.6 \\
\hline Female & 1 & 8.4 \\
\hline Age & & \\
\hline $24-28$ years & 9 & 75.0 \\
\hline 29-33 years & 3 & 25.0 \\
\hline Level of qualification & & \\
\hline B.Pharm/Pharm.D & 7 & 58.3 \\
\hline Master in Pharmacy & 5 & 41.7 \\
\hline Experience & & \\
\hline 1-2 years & 6 & 50 \\
\hline 3-5 years & 6 & 50 \\
\hline Designation & & \\
\hline Staff pharmacist & 6 & 50 \\
\hline Senior pharmacist & 5 & 41.7 \\
\hline Proprietor (Pharmacist) & 1 & 8.4 \\
\hline Type of pharmacy & & \\
\hline Chain & 8 & 66.7 \\
\hline Independent & 33.3 \\
\hline Operating hours of pharmacy & & \\
\hline 24/7 & 8 & 66.7 \\
\hline 16/7 & 4 & 33.3 \\
\hline Antimicrobials dispensed/day & & \\
\hline Mean \pm SD & $47.5 \pm 68.3$ \\
\hline
\end{tabular}

of Pharmacy, University of the Punjab, Lahore, Pakistan (HEC/1000/PUCP/1925A). The identity of the respondents was kept confidential to ensure complete privacy. The anonymity of their opinions was confirmed by using codes by the two researchers (ZS, FKH).

\section{RESULTS}

The demographics of the 12 community pharmacists who were interviewed are given in table 1 . Most of the participants were male $(91.6 \%)$ as females in Pakistan currently do not usually opt to practice in community pharmacies in Pakistan owing to societal reservations; however, one female consented to be interviewed. The respondents were relatively young, with ages ranging from 24 to 33 years, with experience up to 5 years. One practicing pharmacist was the proprietor of the pharmacy as well. The average number of antimicrobial prescriptions dispensed per day by the community pharmacists working in both independent and chain pharmacies were between 30 and 50 prescriptions per day. After TCA, four major themes emerged: (1) knowledge and perception of community pharmacists about antimicrobials, (2) antimicrobial dispensing practices of community pharmacists, 
(3) determinants of AMR, (4) potential interventions to control AMR.

\section{Theme I: Knowledge and perception of community pharmacists about antimicrobials}

During the interviews, the interviewees were asked about their knowledge and perception about antimicrobials. Most of the pharmacists obtained their information about antimicrobials during their undergraduate studies. Only a few pharmacists kept themselves updated through various online resources and other available information.

I had my basic knowledge from study curriculum and I keep myself updated from guidelines, research from the internet and AKU antimicrobial guidelines-the organization where I work [MY]

Basically I have mostly tried to study different antibiotics from different online courses $[\mathrm{M}]$

We have access to a different software like up-to-date, Lexicomp, etc. through which we consult different issues regarding patients and their prescriptions [U]

The majority of pharmacists did not know about any guidelines for antimicrobial use and ASPs. Only one pharmacist had a clear concept about ASP.

I don't have any knowledge about guidelines [A]

Yes I know about IDSA guidelines, but we normally do not follow those guidelines in our practice [Z]

I have heard this terminology quit often but I don't know what it is [Z]

Antimicrobial Stewardship is a program to evaluate the appropriateness of antibiotics and to implement the appropriate use of antibiotics in various organizations $[\mathrm{MY}]$.

Most of the pharmacists knew that antimicrobials should not be dispensed without a prescription. In addition, only a few had information about exact antimicrobial dispensing rules in the Drug Act 1976/DRAP Act 2012 of Pakistan.

I have studied drug law in which schedules $\mathrm{G}$ and $\mathrm{D}$ drugs etc. have been discussed regarding their use. And that these antibiotics should be sold in the presence of a pharmacist only [J]

Well...regarding dispensing of specialized items as antibiotics, we have in Pakistan, schedule G where antibiotics should be sold specifically on prescription from a registered medical practitioner. So, there are certain numbers of antibiotics that shouldn't be sold without a prescription. But the practice is still not much prevalent across Pakistan [MY]

All the pharmacists agreed that AMR was a major problem around the world as well as in Pakistan. All the interviewees also agreed that irrational use of antibiotics was the main reason behind AMR and pharmacist should play their role to prevent irrational antimicrobial use.
Well...because the pharmacist is an important part of the health system from the antimicrobial point of view. The pharmacist knows the chemistry of the drugs as well as about the resistance [M]

In case of community pharmacy setting, we can rationalize only the self-medication particularly for the upper respiratory tract infections, in which case people prefer self-administered medicines. And in case of hospital settings, we can collaborate with other healthcare professionals regarding the appropriate use of the antibiotics [I]

Actually antibiotic resistance is a worldwide problem, not just in our country or region [I]

Well I think the self-medication trend in particular about antibiotics...being used irrationally is the major reason of resistance in our society [I]

\section{Theme Il: Antimicrobials dispensing practices of community pharmacists}

The community pharmacists in Pakistan dispense antimicrobials both with and without prescription. Most of the community pharmacists in this study usually dispense antimicrobials without prescription for common ailments including pharyngitis, sore throats, colds, diarrhoea, tooth ache, influenza, sinusitis, ear infections and also for skin infections. However, a few pharmacists were reluctant to dispense an antimicrobial without a prescription.

No, I never did that. I follow the drug act and don't dispense antimicrobials without prescription [J]

If the patient has a good outcome with previously prescribed item, then I would not resist and I will give that antibiotic [I]

When a poor patient comes to us rather than going to a physician, then as a community pharmacist I help them by giving them antibiotics keeping in mind their cost-effectiveness [K]

If the course is not complete then I dispense antibiotics without prescription as well $[\mathrm{A}]$

Encouraging, the majority of the pharmacists interviewed preferred to refer patients with a critical condition to a physician instead of dispensing antimicrobials.

First I ask them if the patient has a fever, if he has a mild fever then I dispense...otherwise, if he has a high fever or generalized malaise or fatigue as symptoms or sinusitis, then I usually refer him to a physician, I don't usually dispense [FA]

I dispense only when he is in obvious and dire need of an antibiotic. But first I ask him to go to the doctor [A]

Well, as far as a sore throat is concerned, if a patient comes to my pharmacy without a prescription, then, first of all, I ask him to visit a doctor, get a prescription and then I will give you the antibiotic according to that $[\mathrm{W}]$ 
Encouragingly, the community pharmacists interviewed typically counselled their patients about the appropriate dosage regimen of the antibiotic dispensed. They also recommend that the patients manage their mild self-limiting infections without antimicrobials.

Most of the times, I counsel the patients to use house hold remedies for the common cold and upper respiratory tract infections and other infections too, because it's the job of the physician to prescribe [IY]

We have a system that instructions to be given to a patient are added in software and then we print a label of it when dispensed. The label is put in the pack of antibiotic. All the instructions are mentioned on that label according to which we give that antibiotic [U]

Most of the interviewed pharmacists also counselled their patients verbally and a few also gave written instruction on the proper use of any medicine.

Verbally and written too. Patient forgets the verbal instructions...verbal and written both instructions are very necessary $[\mathrm{K}]$

Mostly we try to write the instructions on the label or package of the bottle or sometimes we communicate verbally $[\mathrm{M}]$

The interviewed pharmacists also quite often tried to convince the patients on generic substitution to save costs. However, the acceptability of substitution in Pakistan for any prescribed brand, that is, the originator, is currently limited.

We guide the patient while dispensing that the dispensed medicine is a different brand with the same generic or formula [J]

The patient usually doesn't trust the pharmacist on generic substitution. They usually insist on the medicine prescribed by the doctor on prescription only [FA]

Sometimes there is a good response regarding its cost-effectiveness because we sometimes substitute medicine with a less costly brand [IY].

Sometimes due to unavailability of drugs we go for generic substitution and when sometimes patient cannot afford that specific brands [U]

The community pharmacists are used to dispense antimicrobials both with and without prescription in children, older adults and pregnant females. However, most of the interviewed pharmacists showed concern while dispensing antimicrobials to these populations.

I refer paediatric patients to the children emergencies or children wards or any place like that where they can be treated [W]

I never take risks in case of pregnant women. I always refer them to a specialist for their proper treatment [Z]
For a geriatric or paediatric patient with severe infection, I do not dispense without prescription [K]

I just try to evaluate the patients based on symptoms.

I never go for the age or gender of the patient [M]

\section{Theme III: Determinants of antimicrobial resistance}

One of the main reasons for AMR identified by the interviewees is unnecessary antimicrobial prescribing and dispensing.

Sometimes patients come with 4 antibiotics written on their prescription. Sometimes, patients come with a prescription of 5-6 antibiotics prescribed for chest infection...which I believe is unnecessary $[\mathrm{K}]$

AMR is a big problem because doctors don't go for identification tests of microbes and randomly prescribe antibiotics $[\mathrm{M}]$

Socioeconomic factors are important in determining irrational practices. Most of the time the patients demand a lesser amount of antibiotic than the full prescribed course because they cannot afford the full course.

Well, it depends upon the patient that if he cannot afford the full course of medicine that is written by the doctor, then obviously I have to give him a lesser quantity $[\mathrm{W}]$

Mostly, poor people come to me without prescription who cannot afford to pay the physician. The people in our society are familiar with the thing that a pharmacist is available at the pharmacy we can advise him [K]

The respondents mentioned that most of the patients use leftover antibiotics from their homes that had been prescribed previously to any person within the same family.

Sometimes patients use leftover prescriptions to take antibiotics for the same indication and sometimes they suggest antibiotics to their friends and relatives for certain conditions that I used this antibiotic and got recovered you should also use this [MY]

The patients in Pakistan are like that if one person from a family is prescribed levofloxacin for a upper respiratory tract infection, then when any other family member gets the same disease, patients follows the same prescription so they normally insist to give same antibiotic [Z]

According to a few of the pharmacists interviewed, one of the main reasons for irrational antimicrobial use and subsequent AMR is the business nature of pharmacy. However encouragingly, most of the pharmacists do not have any pressure to sell antimicrobials.

Dispensing antibiotics even without prescription will enhance our sale; output and the owner of pharmacy will also appreciate us" [K] 
In the organization where I work, there is not such pressure from the owners because they appreciate the pharmacist intervening but I have seen a number of cases in local pharmacies where the pharmacy managers do have a pressure on the pharmacists to prescribe the antibiotics to boost up their sales [MY]

In a pharmacy where I am doing the job, a separate medicine rack is assigned to me. I used to dispense medicine including antibiotics for common disease conditions to patients. At the end of the month, I get a good incentive based on the sale [Z]

There was a mixed response of interviewees about the promotional activities by the pharmaceutical company representatives and their visits to pharmacies for the promotion of their products, particularly antibiotics.

I think they have a negative effect. They usually push us to sell their product. I think that is not very good practice [FA]

The visit of the sales representatives at community pharmacies is not so influential to increase the sale of antimicrobials [MY]

Mostly, the sale representatives don't approach the pharmacist, but the doctors [K]

\section{Theme IV: Potential interventions to control antimicrobial resistance}

Interviewees typically agreed that we cannot control AMR without the implementation of laws and legislation for antibiotic use. The interviewed pharmacists also pointed out a few strategies to control the emergence of AMR by involving all key stake holders of the healthcare system including physicians, nurses and pharmacists.

At the individual level, we cannot play a special role to overcome this problem. Every sector of the healthcare should participate in its role [I]

For this, a proper guideline and a country-wide policy should be made. Data should be collected, problems should be identified which should be faced steadily [U]

First of all, we need to conduct certain educational seminars and awareness programs for the healthcare professionals and this does not only include the pharmacists but also the doctors, nurses and the paramedical staff $[\mathrm{MY}]$

The community pharmacists also highlighted restriction strategies to control the misuse of antimicrobials.

We can reduce the risk of antimicrobial resistance by limiting the overprescribing of antibiotics for unlabeled indications $[\mathrm{A}]$

Like narcotics and other control drugs, antibiotics should also be controlled to dispense without prescription and the proper record should be maintained $[\mathrm{Z}]$
All the pharmacists agreed that conferences, workshops and continuous education programmes could play a significant role to overcome the threat of AMR. However, they themselves typically didn't attend any such event related to antimicrobials.

Yes definitely, one doesn't get to know everything at once. Learning is a continuous process. So, to update our knowledge a pharmacist should attend every kind of conferences and seminars to increase and enhance his previous knowledge [J]

I totally agree that such conferences should be held especially for fresh graduates who don't know much about dosage and indications for which antibiotics are used [K]

I didn't attend any workshop in the past one year because no such workshop was arranged [B]

I attended a workshop in PC by GSK on minimizing the use of antibiotics, avoiding new generation antibiotics to stop resistance [IY]

As healthcare professionals, pharmacists are also getting recognition as a healthcare provider and patients also come to pharmacists for consultation. Consequently, community pharmacists should be involved in the national action plan of Pakistan on AMR.

When we tell them that we are pharmacist, they trust us knowing that we are qualified and literate [J]

Patients of the elite class are satisfied mostly. They are familiar with the pharmacists $[\mathrm{Y}]$

Health authorizes should recognize the role of community pharmacists as healthcare provider if they want to fight against antimicrobial resistance [I]

Pharmacist should be given a proper role which nowadays they don't have [U]

\section{DISCUSSION}

In order to manage infectious diseases appropriately in the future, the judicious use of antimicrobials is essential. In view of their responsibility for rationalising medication use, pharmacists are critically placed, especially in LMICs to enhance the appropriate use of antimicrobials to reduce AMR, which is increasingly recognised across countries. ${ }^{28}$ This is particularly important in Pakistan where antimicrobials can easily be purchased without a prescription as most pharmacies do not comply with the legal requirements. ${ }^{29} 30$ This is illustrated in this study where most of the interviewed community pharmacists felt comfortable dispensing antimicrobials without a doctor's prescription, similar to studies conducted among LMICs as well as Saudi Arabia where antimicrobial dispensing without doctor's prescription is common. ${ }^{78}{ }^{20}$ Contrary to a recent study regarding the knowledge of community pharmacist of Pakistan about antimicrobials, our study highlighted that pharmacists had poor knowledge about antimicrobials, ASPs and related antimicrobial 
guidelines. ${ }^{31}$ However, our findings are similar to the findings of Shahid $e t$ al where there were concerns with the knowledge and attitude of healthcare professionals towards $\mathrm{AMR}^{32}$ as well as among pharmacy students and community pharmacies in other countries. ${ }^{10} 33$ There are also concerns regarding the knowledge of ASPs among pharmacy students in South Africa. ${ }^{16}$

According to the interviewees, mostly of the patients purchased antimicrobials without a prescription for upper respiratory tract infections (URTIs) including coughs, colds and sore throats as well as for gastrointestinal infections. However, the tendency to dispense antimicrobials without prescription in the case of a simple infection is not sending out the appropriate message to patients or parents and endorsing their continued self-purchasing. ${ }^{34}$ Such practices show that the current legislation in Pakistan is not being effectively enforced to influence the decision of pharmacists in dispensing antimicrobials without a prescription from physicians. These factors could increase the risk of emergence of multidrug-resistant microbes. ${ }^{35}$ Similar to other LMICs, most of the interviewees in our study observed that behavioural and socioeconomic factors including self-medication, use of leftover antimicrobials, non-adherence to the prescribed or dispensed course, lack of healthcare facilities and avoidance of a consultation fee due to poverty, resulted in the irrational use of antimicrobials. ${ }^{36} 37$ Similar to other published studies, patients often do not buy the full course of antimicrobials and discontinue them when they feel better. ${ }^{38}$ In addition similar to other studies, speed of recovery and financial incentives to physicians also appear to be driving factors of irrational antimicrobial prescribing. ${ }^{39}$ Monetary incentives and the business orientation of pharmacies in Pakistan also appear to enhance irrational antibiotic dispensing, which need further investigation in Pakistan. The enforcement of the law prohibiting the sale of antimicrobials without a prescription from a registered medical practitioner should decrease the total volume of antimicrobials used as seen in other countries. ${ }^{13}$ However, this has to be balanced against issues of affordability, especially if patients cannot afford to see both a physician and purchase their medicines. In addition, issues of physician availability, in particular in more rural areas of Pakistan, and manpower issues involved with effectively monitoring such practices especially with the growing number of pharmacies in Pakistan.

Consequently, overall we believe the training of community pharmacists about rational antimicrobial use, especially for predominantly viral infections would be very valuable in Pakistan, and an appropriate way forward building on the situation in other LMICs. ${ }^{13}$ This is endorsed by interviewees' willingness to learn about the rational use of antimicrobials. However, the interviewees believed that the promotion of the rational use of antimicrobials is not possible without the involvement of relevant organisations and authorities. They also pointed out that enforced regulatory action from concerned organisations is required. Consequently, in order to rationalise the use of antimicrobials in Pakistan, a multifaceted strategy needs to be implemented involving all key stakeholder groups including physicians, pharmacists, patients and policy-makers. Community pharmacists can also be involved in ASPs to further enhance the appropriate use of antimicrobials in ambulatory care, which is endorsed internationally and being enacted in other countries. ${ }^{40}$ Currently in Pakistan, community pharmacies have no established ASPs; however, they are increasingly aware of their role as antimicrobial stewards. ${ }^{31}$ Consequently, community pharmacists should be encouraged to follow national and international guidelines about antimicrobial use and dispensing, starting with additional training in Pharmacy schools and continued with further educational initiatives once qualified using the internet and other media. This is because our current study highlights that most pharmacists in Pakistan hardly participate in any antimicrobial stewardship campaigns as seen by high rates of self-purchasing in Pakistan. This is a concern as we have seen in other LMICs that there is little dispensing of antibiotics for URTIs in pharmacies allied to universities. ${ }^{14}$ Nevertheless, encouragingly there were some clinical conditions where interviewees were unwilling to dispense antimicrobials without a prescription including paediatric patients and pregnant women. However, against this, only a few community pharmacists verified patients' medical history before dispensing antimicrobials, which is a concern, especially with potential allergies. Dispensing of an antimicrobial for predominantly viral infections such as a cough, cold or sore throat also increases AMR rates. ${ }^{6}$ There was also no appropriate follow-up protocol and consultation related to the management of common side effects. These issues need to be addressed if Pakistan is to reduce its AMR rate, and this will be followed up in the future. Alongside this, there needs to be additional education for patients and carers to help reduce the pressures on physicians and pharmacists to prescribe or dispense an antibiotic, especially for self-limiting conditions such as coughs and colds. ${ }^{27}$

\section{Limitations}

A limitation of this survey is that community pharmacists who decided not to take part may have opinions significantly different from those of responders, probably leading to unmeasured bias. However, we believe these limitations were minimised using our comprehensive approach including additional interviews to ensure we had explored all situations. Another possible limitation of this study is the inclination of the interviewee to give socially desirable answers instead of revealing their true opinions. We believe this problem was also minimised by assuring pharmacists complete privacy. Overall, we believe the results of this first qualitative study are promising and give a good overview of the current extent of dispensing of antibiotics in Pakistan without a prescription as well as knowledge regarding antimicrobials and AMR and potential ways to start addressing identified concerns. 


\section{CONCLUSION}

We believe our findings indicate that irrational antimicrobial dispensing and use of antimicrobials are common among community pharmacists in Pakistan owing to a lack of knowledge combined with commercial and other pressures. Overall, the community pharmacists perceived that behavioural and societal environments contributed to irrational antimicrobial prescribing and dispensing and the subsequent development of AMR. The interviewees suggested a need for a multidisciplinary framework and activities to improve antimicrobial use and prevent AMR. This includes additional education of physicians, pharmacists, the public, social scientists, government agencies and non-profit organisation antimicrobials about AMR and the aetiology of self-limiting diseases such as URTIs. This along with the proper implementation and enforcement of strict legislation at all levels of the drug distribution system, where appropriate, can reduce the burden of AMR. There also needs to be improvements in the licensing of generic medicines to address concerns among physicians, pharmacists and patients to reduce affordability as a barrier to taking a full course of antibiotics or, alternatively, seeing a physician first. These activities will be followed up in the future as Pakistan progresses with its National Action plan to reduce AMR rates.

Contributors Conceptualisation: ZS, MH. Methodology: ZS, FS. Formal analysis: ZS, FH. Writing —original draft: ZS. Writing—review and editing: BG, FH. Approval of final manuscript: all authors.

Funding The authors have not declared a specific grant for this research from any funding agency in the public, commercial or not-for-profit sectors.

Competing interests None declared.

Patient consent for publication Not required.

Provenance and peer review Not commissioned; externally peer reviewed.

Data availability statement There are no data in this work.

Open access This is an open access article distributed in accordance with the Creative Commons Attribution Non Commercial (CC BY-NC 4.0) license, which permits others to distribute, remix, adapt, build upon this work non-commercially, and license their derivative works on different terms, provided the original work is properly cited, appropriate credit is given, any changes made indicated, and the use is non-commercial. See: http://creativecommons.org/licenses/by-nc/4.0/.

ORCID iD

Zikria Saleem http://orcid.org/0000-0003-3202-6347

\section{REFERENCES}

1. Saleem Z, Hassali MA, Hashmi FK. Pakistan's National Action Plan for antimicrobial resistance: translating ideas into reality. Lancet Infect Dis 2018;18:1066-7.

2. Cosgrove SE. The relationship between antimicrobial resistance and patient outcomes: mortality, length of hospital stay, and health care costs. Clin Infect Dis 2006;42(Suppl 2):S82-9.

3. Founou RC, Founou LL, Essack SY. Clinical and economic impact of antibiotic resistance in developing countries: a systematic review and meta-analysis. Plos One 2017;12:e0189621.

4. Saleem Z, Hassali MA. Travellers take heed: outbreak of extensively drug resistant (XDR) typhoid fever in Pakistan and a warning from the US CDC. Travel Med Infect Dis 2019;27.

5. Costelloe C, Metcalfe C, Lovering A, et al. Effect of antibiotic prescribing in primary care on Antimicrobial resistance in individual patients: systematic review and meta-analysis. BMJ 2010;340:c2096.

6. Llor C, Bjerrum L. Antimicrobial resistance: risk associated with antibiotic overuse and initiatives to reduce the problem. Ther Adv Drug Saf 2014;5:229-41.
7. Auta A, Hadi MA, Oga E, et al. Global access to antibiotics without prescription in community pharmacies: a systematic review and meta-analysis. J Infect 2019;78:8-18.

8. Hadi MA, Karami NA, Al-Muwalid AS, et al. Community pharmacists' knowledge, attitude, and practices towards dispensing antibiotics without prescription (DAwP): a crosssectional survey in Makkah Province, Saudi Arabia. Int J Infect Dis 2016;47:95-100.

9. Mbonye AK, Buregyeya E, Rutebemberwa E, et al. Prescription for antibiotics at drug shops and strategies to improve quality of care and patient safety: a cross-sectional survey in the private sector in Uganda. BMJ Open 2016;6:e010632.

10. Hoxha I, Malaj A, Kraja B, et al. Are pharmacists' good knowledge and awareness on antibiotics taken for granted? The situation in Albania and future implications across countries. J Glob Antimicrob Resist 2018;13:240-5.

11. Sahoo KC, Tamhankar AJ, Johansson E, et al. Antibiotic use, resistance development and environmental factors: a qualitative study among healthcare professionals in Orissa, India. BMC Public Health 2010;10.

12. Bahnassi A. A qualitative analysis of pharmacists' attitudes and practices regarding the sale of antibiotics without prescription in Syria. Journal of Taibah University Medical Sciences 2015;10:227-33.

13. Markovic-Pekovic V, Grubisa N, Burger J, et al. Initiatives to reduce nonprescription sales and dispensing of antibiotics: findings and implications. J Res Pharm Pract 2017;6:120-5.

14. Mukokinya MMA, Opanga S, Oluka M, et al. Dispensing of antimicrobials in Kenya: a cross-sectional pilot study and its implications. J Res Pharm Pract 2018;7:77-82.

15. Rezal RS, Hassali MA, Alrasheedy AA, et al. Prescribing patterns for upper respiratory tract infections: a prescription-review of primary care practice in Kedah, Malaysia, and the implications. Expert Rev Anti Infect Ther 2015;13:1547-56.

16. Burger M, Fourie J, Loots D, et al. Knowledge and perceptions of antimicrobial stewardship concepts among final year pharmacy students in pharmacy schools across South Africa. S Afr J Infect Dis 2016;31:84-90.

17. I KA A. Self-medication of antibiotics: a perspective on alarming situation in Peshawar, Khyber Pakhtunkhwa, Pakistan. Archives of Pharmacy Practice 2015;6:68-70.

18. Bilal M, Haseeb A, Khan MH, et al. Self-medication with antibiotics among people Dwelling in rural areas of Sindh. J Clin Diagn Res 2016;10:OC08-13.

19. Aziz MM, Masood I, Yousaf M, et al. Pattern of medication selling and self-medication practices: a study from Punjab, Pakistan. Plos One 2018;13:e0194240.

20. Al-Faham Z, Habboub G, Takriti F. The sale of antibiotics without prescription in pharmacies in Damascus, Syria. J Infect Dev Ctries 2011;5:396-9.

21. Kaae S, Malaj A, Hoxha I. Antibiotic knowledge, attitudes and behaviours of Albanian health care professionals and patients - a qualitative interview study. J Pharm Policy Pract 2017;10.

22. Kotwani A, Wattal C, Joshi PC, et al. Irrational use of antibiotics and role of the pharmacist: an insight from a qualitative study in New Delhi, India. J Clin Pharm Ther 2012;37:308-12.

23. Tong A, Sainsbury P, Craig J. Consolidated criteria for reporting qualitative research (COREQ): a 32-item checklist for interviews and focus groups. Int J Qual Health Care 2007;19:349-57.

24. FdS DF, Barberato-Filho S, Lopes LC, et al. Assessment of Brazilian pharmacists' knowledge about antimicrobial resistance. The Journal of Infection in Developing Countries 2015;9:239-43.

25. Bojanić L, Marković-Peković V, Škrbić R, et al. Recent initiatives in the Republic of Srpska to enhance appropriate use of antibiotics in ambulatory care; their influence and implications. Front Pharmacol 2018;9.

26. Alabid AHMA, Ibrahim MIM, Hassali MA. Antibiotics dispensing for URTIs by community pharmacists (CPS) and general medical practitioners in Penang, Malaysia: a comparative study using simulated patients (SPS). J Clin Diagn Res 2014;8:119-23.

27. Godman B, Fadare J, Kibuule D, et al. Initiatives Across Countries to Reduce Antibiotic Utilisation and Resistance Patterns: Impact and Implications. In: Arora G, Sajid A, Kalia VC, et al, eds. Drug resistance in bacteria, fungi, malaria, and cancer. Cham: Springer International Publishing, 2017: 539-76.

28. Howard P, Ashiru-Oredope D, Gilchrist M. Time for pharmacy to unite in the fight against antimicrobial resistance. Stroke 2018;13.

29. Sturm AW, van der Pol R, Smits AJ, et al. Over-the-counter availability of antimicrobial agents, self-medication and patterns of resistance in Karachi, Pakistan. J Antimicrob Chemother 1997;39:543-7. 
30. Hussain A, Ibrahim MIM, Baber Z-ud-D. Compliance with legal requirements at community pharmacies: a cross sectional study from Pakistan. Int J Pharm Pract 2012;20:183-90.

31. Sarwar Mrehan, Saqib A, Iftikhar S, et al. Knowledge of community pharmacists about antibiotics, and their perceptions and practices regarding antimicrobial stewardship: a cross-sectional study in Punjab, Pakistan. Infection and drug resistance 2018;11:133-45.

32. Shahid A, Iftikhar F, Arshad MK, et al. Knowledge and attitude of physicians about antimicrobial resistance and their prescribing practices in services Hospital, Lahore, Pakistan. JPMA 2017;67.

33. Ahmad A, Khan MU, Moorthy J, et al. Comparison of knowledge and attitudes about antibiotics and resistance, and antibiotics selfpracticing between Bachelor of pharmacy and doctor of pharmacy students in southern India. Pharm Pract 2015;13.

34. Al-Hamad A. Over-the-counter delivery of antibiotics: are we sending the right message? Am J Infect Control 2012;40:81-2.
35. Tillotson GS, Zinner SH. Burden of antimicrobial resistance in an era of decreasing susceptibility. Expert Rev Anti Infect Ther 2017;15:663-76.

36. Kristiansson C, Grape M, Gotuzzo E, et al. Socioeconomic factors and antibiotic use in relation to antimicrobial resistance in the Amazonian area of peru. Scand J Infect Dis 2009;41:303-12.

37. Saleem Z, Saeed H, Ahmad M, et al. Antibiotic Self-Prescribing trends, experiences and attitudes in upper respiratory tract infection among pharmacy and Non-Pharmacy students: a study from Lahore. PLoS One 2016;11:e0149929.

38. Hawkings NJ, Butler CC, Wood F. Antibiotics in the community: a typology of user behaviours. Patient Educ Couns 2008;73:146-52.

39. Reynolds L, McKee M. Factors influencing antibiotic prescribing in China: an exploratory analysis. Health Policy 2009;90:32-6.

40. MacDougall C, Polk RE. Antimicrobial stewardship programs in health care systems. Clin Microbiol Rev 2005;18:638-56. 